\title{
La S.O.T.EST, pour quoi faire?
}

\author{
F. Demigneux
}

Polyclinique Montier la Celle, 17, rue Baltet, F-10120 Saint-André-1es-Vergers

Parisien d'origine, Blésois dans ma jeunesse, ayant appris la Chirurgie Orthopédique à Paris chez des maîtres qui s'appelaient Padovani, Méary, ou qui s'appellent toujours Anne Languepin et Jacques Duparc, je me suis un jour installé à Troyes par hasard et par l'amitié d'un aubois qui se vouait aussi à la Chirurgie. Qui suis-je donc et au nom de quoi pourrai-je convaincre des Strasbourgeois, des Nancéiens, des Dijonnais ou des Belfortains qu'il faut s'investir dans la S.O.T.EST ?

Crée à l'origine par les Chefs des Services d'Orthopédie des capitales régionales de 1'Est pour offrir à leurs élèves et à eux-mêmes un lieu d'échange et une tribune pour la présentation de leurs travaux dans un cadre moins formel et plus libre que la SO.F.C.O.T., cette Société a vécu de belles années. Pour le parisien que j'étais, installé en limite de cette grande région et encore relié à l'Hôpital Bichat, ma "maison mère", par un cordon ombilical solide, cette société restait lointaine. De l'extérieur, elle m'inspirait une idée d'exotisme froid et de mandarinat, peu compatible avec mon esprit d'indépendance. J'allais plutôt aux réunions de la Société d'Orthopédie de l'Ouest, dans laquelle il $y$ avait bon nombre d'anciens parisiens, plus ouverte aux chirurgiens libéraux. C'est à l'Ouest que je fis la connaissance d'Yves
Gérard qui finit par me convaincre d'organiser une réunion de la S.O.T.EST à Troyes. Pour me mettre dans l'ambiance j'assistais à deux réunions de la S.O.T.EST et je n'en ai manqué aucune depuis lors. J'y ai trouvé de bonnes amitiés, des régions et des gens à découvrir, et peut-être un sentiment d'utilité auquel je n'ose pas me soustraire !

Dans les dernières années les réunions orthopédiques se sont multipliées au fur-et-à-mesure que la spécialité se subdivisait : orthopédie pédiatrique, chirurgie de la main, arthroscopie, sans parler des réunions consacrées au rachis, au genou, à l'épaule, au pied, etc... Ces réunions sont irremplaçables car elles apportent sur un sujet bien ciblé le maximum d'enseignement dans le minimum de temps. les grandes réunions comme la SO.F.C.O.T. n'en souffrent pas car elles sont capables de mobiliser sur une variété de sujets les meilleurs spécialistes dans un site privilégié. Mais nous n'y allons pas tous, tous les ans, et pour toute la durée du congrès. Les congrès lointains sont bien fréquentés aussi, plus l'hiver que l'été. Au mois de juin les réunions tombent les unes sur les autres. On ne peut aller partout ; il faut choisir. Les membres de la S.O.T.EST qui ne participent pas à la réunion annuelle de leur Société ne sont pas accusés de pantou- flage : ils sont tous supposés s'instruire ailleurs!

Le problème est que leurs absences cumulées vident notre congrès d'une partie de sa substance, réduisent l'auditoire des orateurs qui se sentent frustrés, posent des questions aux fabricants qui ont apporté leur soutien financier et ne sont pas payés de retour, vexent l'organisateur qui s'est donné de la peine et détournent celui qui est venu pour la première fois de revenir l'année suivante. Alors les membres du Bureau, qui ne retirent de leur fonction aucun avantage, je vous l'assure, se posent la question de savoir ce qu'il faudrait faire pour que les 200 membres de la S.O.T.EST, qui payent une cotisation non symbolique, témoignent vis-à-vis de leur Société, du minimum d'intérêt que représenterait leur présence à cette réunion annuelle. Ils rêvent d'une S.O.T.EST qui réunirait chaque année, dans un lieu qui n'est jamais géographiquement bien éloigné, tous les chirurgiens orthopédistes ou intéressés par l'orthopédie, de Strasbourg à Besançon, de Charleville-Mézières à Mulhouse, de Reims à Belfort, de Dijon à Colmar, de Troyes à Thionville, en passant par Nancy, Metz, Verdun, Bar-le-Duc, Châlons, Vittel, Chaumont, Vesoul, Epinal ... sans oublier Toul, Longwy, Sarreguemines, Pont-à-Mousson, SaintDié, Epernay, Vitry-le-François, 
Langres... Rassurez-vous : je m'arrête ! Mais rendez-vous compte : pour peu que viennent aussi tous les Chefs de Clinique qui ne sont pas de garde à Strasbourg, Reims, Nancy, Dijon et Besançon, cela ferait du monde ! Et les membres du Bureau relisent Alphonse Daudet dont le curé de Cucugnan avait trouvé le moyen de ramener ses ouailles dans le droit chemin, en espérant y trouver une source d'inspiration !

Certes il faut que l'organisateur de la réunion - si l'on est modeste -, du congrès, - si l'on a de l'ambition -, remplisse le cahier des charges : une date connue suffisamment longtemps à l'avance, qui ne coïncide pas avec une autre réunion de première grandeur, des sujets intéressants, un lieu convenable, des orateurs que l'on a envie d'écouter. Il faut que l'organisation soit amicale et structurée en même temps et qu'il reste $\mathrm{du}$ temps pour la discussion. Il est nécessaire qu'il sache ménager des temps de rencontre dans un cadre agréable propice à la détente et à la découverte. Lorsqu'il a accompli ce travail, car c'en est un, et qui prend du temps, il serait juste qu'il en soit remercié par la présence des membres de la Société pour le compte de laquelle il l'a fait. Et comme la vie civilisée est un échange de bons procédés, il remerciera ceux qui ont contribué au succès de sa réunion en se faisant un devoir de répondre à leur invitation lorsque ce sera eux qui supporteront la charge de l'organisation.

Certes il serait prétentieux de dire que manquer notre réunion annuelle constituerait pour chacun d'entre nous un déficit culturel dont il ne se remettrait pas. Il serait tout aussi prétentieux de croire qu'il n'y a rien à apprendre à la S.O.T.EST. Comme il serait prétentieux pour un chirurgien, quelque soit son expérience, son âge et son talent, de ne plus fréquenter que les réunions dans lesquelles il est invité à parler. Nous exerçons un noble artisanat dans lequel ceux qui ont défriché un domaine où ils ont acquis leur notoriété, sont souvent, dans un autre domaine débiteurs des autres. Et c'est bien ainsi.

Mais il y a autre chose à découvrir dans notre Société : de nouvelles amitiés, des affinités à portée de voiture, des régions diverses, méconnues, attachantes, insoupçonnées et proches ; des hauts-lieux cachés à l'écart des autoroutes. Enfin ces réunions peuvent être un lieu privilégié de rencontre : bavarder à 1'occasion du déjeuner ou du dîner avec tel maître français ou étran- ger hors de tout protocole, prendre des contacts en vue d'une visite, d'une installation ou d'une succession, faire connaissance avec un confrère nouvellement installé, intégrer les internes et les Chefs de Clinique dans ce milieu professionnel de la Chirurgie Orthopédique en leur donnant de la profession une vision empreinte de sérieux, de confraternité, de partage et d'humanisme.

Ces objectifs ne seront atteints que si les Chefs des Services d'Orthopédie et de Traumatologie ont le sentiment que la S.O.T.EST est un instrument utile à la formation de leurs internes et les font travailler pour présenter des travaux; que si les jeunes chirurgiens se sentent chez eux dans cette Société et veulent la faire vivre ; que si chacun accepte de se déplacer pour écouter les autres même s'il ne parle pas ; que si l'on croit à la situation privilégiée de notre région, proche de la Belgique, de l'Allemagne et de la Suisse, comme chance de développement à cultiver ; que si l'on pense qu'il faut que cette Société vive.

Vivre, c'est grandir, se développer, se renforcer, se structurer, s'enrichir. Végéter n'offre aucun intérêt. 